Published in "Behavioural Processes 90(3): 364-371, 2012"

which should be cited to refer to this work.

\title{
Recognition memory in tree shrew (Tupaia belangeri) after repeated familiarization sessions
}

\author{
Abbas Khani ${ }^{a, b}$, Gregor Rainer ${ }^{a, b, *}$ \\ a Visual Cognition Laboratory, Department of Medicine, CH 1700 Fribourg, Switzerland \\ b Center for Cognition, University of Fribourg, CH 1700 Fribourg, Switzerland
}

\begin{abstract}
Recognition memories are formed during perceptual experience and allow subsequent recognition of previously encountered objects as well as their distinction from novel objects. As a consequence, novel objects are generally explored longer than familiar objects by many species. This novelty preference has been documented in rodents using the novel object recognition (NOR) test, as well is in primates including humans using preferential looking time paradigms. Here, we examine novelty preference using the NOR task in tree shrew, a small animal species that is considered to be an intermediary between rodents and primates. Our paradigm consisted of three phases: arena familiarization, object familiarization sessions with two identical objects in the arena and finally a test session following a 24-h retention period with a familiar and a novel object in the arena. We employed two different object familiarization durations: one and three sessions on consecutive days. After three object familiarization sessions, tree shrews exhibited robust preference for novel objects on the test day. This was accompanied by significant reduction in familiar object exploration time, occurring largely between the first and second day of object familiarization. By contrast, tree shrews did not show a significant preference for the novel object after a one-session object familiarization. Nonetheless, they spent significantly less time exploring the familiar object on the test day compared to the object familiarization day, indicating that they did maintain a memory trace for the familiar object. Our study revealed different time courses for familiar object habituation and emergence of novelty preference, suggesting that novelty preference is dependent on well-consolidated memory of the competing familiar object. Taken together, our results demonstrate robust novelty preference of tree shrews, in general similarity to previous findings in rodents and primates.
\end{abstract}

\section{Introduction}

Tree shrews (Tupaia belangeri) are day active mammals from Southeast Asia and are considered as intermediates between rodents and primates (Fuchs and Flügge, 2002; Martin, 1968; Petry et al., 1984). Genetic information is available, since the tree shrew was selected as one of 25 mammals for low coverage sequencing by the Broad Institute (http://www.broadinstitute.org/mammals). They are small slender mammals roughly the size of a rat with a long bushy tail, and exhibit agile and quick movements in arboreal environments (Lu and Petry, 2003). The small size of the animal combined with its close proximity to primates from an evolutionary point of view, uniquely places the tree shrew as an invaluable candidate for many behavioral tests. Nonetheless, the behavioral studies done with tree shrews have been rather

* Corresponding author at: Visual Cognition Laboratory, Department of Medicine, Chemin du Musee 5, 1700 Fribourg, Switzerland. Tel.: +41263008689.

E-mail address: gregor.rainer@unifr.ch (G. Rainer). limited when compared to more standard animal models such as the rat or non-human primate. One line of work has established tree shrews as a model animal for social stress (Fuchs, 2005; Fuchs and Flügge, 2002, 2003; Kozicz et al., 2008; Zambello et al., 2010). Other aspects of tree shrew behavior such as learning set formation (Ohta et al., 1985), visuo-spatial learning (Ohl et al., 1998; Ohl and Fuchs, 1999) and transitive inference (Takahashi et al., 2008) have also been investigated. In general, these studies show that tree shrews have competent cognitive abilities. For instance, all participating tree shrews were able to learn an inference task, whereas only a subset of rats achieved criterion performance on the same task (Takahashi et al., 2008). In addition, there has been some work on visually based behaviors (Callahan and Petry, 2000; Lu and Petry, 2003; Petry et al., 1984; Raczkowski et al., 1976), since tree shrews-unlike rodents - have a well developed visual system and can be readily trained to perform various visually based tasks. Given this well-developed and highly differentiated visual system, our aim was to extend the repertoire of tree shrew visual behavioral paradigms by examining their behavior in an object recognition memory test. 
Since its introduction (Ennaceur and Delacour, 1988), the novel object recognition (NOR) test has gained popularity as a recognition memory test in rodents. It consists of an arena familiarization, an object (usually a pair of identical objects) familiarization and, after a certain interval, a test phase. The NOR test has been used to investigate the influence of a broad range of manipulations on memory (Aubele et al., 2008; Fahey et al., 2008; Jurdak and Kanarek, 2009; Lockrow et al., 2010; Reger et al., 2009; Weible et al., 2009). This simple test is based on the premise that rodents inherently tend to preferentially explore a novel object in the presence of an already familiar object. This novelty preference necessitates an intact recognition memory of the previously seen familiar object. The NOR test has several advantages over other memory tests including the absence of any externally applied reinforcement or rule, making it particularly suitable for assessing recognition memory. According to the interval between object familiarization and test phases, typically spanning from one minute to over $24 \mathrm{~h}$, the task is suited for examination of both short term memory (STM, 1-90 min interval) and long term memory (LTM, usually $24 \mathrm{~h}$ interval). Generally, rodents do not show significant novelty preference in the long term $(24 \mathrm{~h}$ ) memory test in the NOR task (Ennaceur and Delacour, 1988; Okuda et al., 2004; Roncarati et al., 2009; Tellez et al., 2010), with some notable exceptions (Botton et al., 2010; Reger et al., 2009). The possible reasons for largely absent LTM in rodents in the NOR paradigm have not been discussed extensively in previous literature.

The weak novelty preference observed after $24 \mathrm{~h}$ in rodents is thought to be due to a failure to maintain a memory of the familiar object over this period. This poor LTM provides an opportunity for testing memory enhancers (Okuda et al., 2004; Wong et al., 2010; Roncarati et al., 2009). By contrast, rodents do not provide a good model for assessing detrimental effects on LTM. Nevertheless, to date, almost all NOR tests have been done in rodents and this test is increasingly used in rodent animal models of various diseases including Alzheimer's Disease (Donkin et al., 2010; Greco et al., 2010; Hillen et al., 2010; Lu et al., 2009; Mouri et al., 2007; Nishida et al., 2006; Taglialatela et al., 2009), schizophrenia (Hauser et al., 2009; McLean et al., 2009; Neill et al., 2010; Ozawa et al., 2006; Powell et al., 2007; Vigano et al., 2009), and Down's syndrome (Lockrow et al., 2010). The absence of any external reinforcement in the NOR test is particularly important as it makes it more comparable to memory tests in human, which are normally conducted without the use of positive or negative reinforcers such as food and electric shock (Ennaceur and Delacour, 1988). Additionally, validating the NOR task in an animal model that is phylogenetically closer to humans could strengthen the extrapolation of animal studies to our understanding of human cognitive functions, as well as facilitate translational medicine in memory-related complications.

Here, we used the NOR task in tree shrew and showed robust novelty preference following three, but not one day of object familiarization. In addition, we found that exploration time for familiar objects was already reduced following a single object familiarization session. Our study provides novel insights into the dynamics of recognition memory formation in the tree shrew.

\section{Materials and methods}

\subsection{Animals}

Adult male and female tree shrews were housed in large cages that were connected through a tube to a nesting box. A total of 13 animals ( 4 males) aged 1-5 years old were used in the experiments. They were kept in a temperature controlled room and maintained on a standard 12-h light/dark cycle (light on at 06:00). Food and water were available ad libitum. All animals were naïve to the current test and had no prior experience in any behavioral test. All experimental procedures were in compliance with European (ECVD 86/609/EEC) and applicable Swiss regulations.

\subsection{Apparatus}

Our testing apparatus was composed of an arena with a camera mounted on the top of the arena. The arena was designed to resemble animals' cage thereby minimizing animals' stress and maximizing validity of the experiment. The arena was an openfield box (length and width; $60 \mathrm{~cm} \times 60 \mathrm{~cm}$ ) covered by a pyramidal ceiling at the top. The camera was mounted at a height of about $70 \mathrm{~cm}$. Arena walls were made of dark-grey-painted wood and the arena was illuminated with LED lights fixed on the ceiling. The arena was placed in a quiet testing room close to the animal room. The objects to be remembered were objects made of Lego (approx. $5 \mathrm{~cm} \times 10 \mathrm{~cm} \times 10 \mathrm{~cm})$ in experiments 1 and 2 and metal soft drink cans (red with white writing and logo on it; approx. $6 \mathrm{~cm}$ diameter $\times 12 \mathrm{~cm}$ height) and a white plastic container with a red lid (approx. $7 \mathrm{~cm} \times 7 \mathrm{~cm} \times 10 \mathrm{~cm}$ ) in experiment 2 .

\subsection{Procedure}

Experiment 1. The experiment consisted of three phases: Arena familiarization; Object familiarization; and Test phase. In all phases, every animal was carried inside the nesting box to the test room which was connected to the arena using a flexible tube. The gate was opened and the animals were given the opportunity to enter and explore the arena. In all phases and in all experiments, the animals were able to freely commute between the arena and the nesting box through the connecting tube. This allowed them to return to a familiar and safe place after exploration. At the end of the experiment, the gate was closed while the animals were in the nesting box, and then animals were carried back to their cage. Pilot experiments showed that tree shrews require a lot of time to get familiar to the arena. The arena familiarization trials were considered complete when 10 min elapsed since the first time the animal had entered the arena.

After three days of familiarization to the empty arena, two identical objects were included in the arena in the object familiarization phase. Again animals could commute freely between the arena and the nesting box and now explore objects inside the arena. Every animal was allowed five minutes (starting when the animal first entered the arena) to explore objects in a single object familiarization session and video was recorded for every animal. After all trials, the arena and objects were cleaned by $70 \%$ ethanol to avoid any olfactory trails. The animals were subsequently returned to their home cage and after a 24-h retention period, one of the objects was exchanged with a novel object, and tree shrews were again brought to the arena and were allowed to explore the familiar and novel objects during the test phase. Familiar and novel objects used in this experiment were made of Lego and were counterbalanced for different animals. Every animal was allowed five minutes to navigate in the arena and freely explore objects while their behavior was recorded by the camera. Fig. 1 shows the navigation map of a tree shrew superimposed on the arena with respective objects during object familiarization and test phases. It should be noted that all phases of all experiments were conducted in a closed system and the experimenter did not handle animals or otherwise intervene during the experiment. The experimenter was monitoring animal behavior online on a computer screen through the connected camera.

Experiment 2. This experiment examined how retention is boosted by repeated exposure to familiar objects. It was conducted one month after the end of experiment 1 , and was similar to the first experiment except that every animal had just one day of 

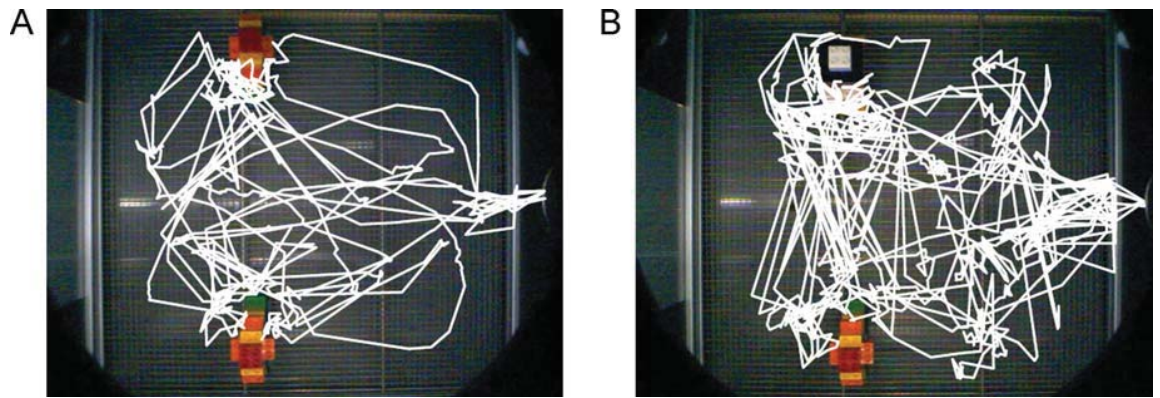

Fig. 1. Tracks of movements of a tree shrew during object familiarization and test phases superimposed on the image of the arena with respective objects. (A) The tracing of the animal's movements during an object familiarization phase shows a similar amount of exploration of both identical objects. (B) The animal spent more time near the novel object (top) compared to the familiar object (bottom).

arena familiarization followed by three subsequent days of object familiarization with one session per day. The time spent exploring each object and the total object exploration time on every object familiarization day and on the test day were calculated for every animal. This experiment was repeated twice with different sets of objects. To distinguish between these two repetitions, hereinafter we will refer to the first one (soft drink can and plastic bottle as objects) as experiment $2 \mathrm{a}$ and the second one as experiment $2 \mathrm{~b}$ (objects made of Lego). There was a three week interval between the experiments $2 \mathrm{a}$ and $2 \mathrm{~b}$. In addition to five animals that took part in experiment 1 , two more animals were included in experiment 2a (but one in experiment 2b). As in experiment 1, familiar and novel objects were counterbalanced among animals in each experiment.

Control experiment. We counterbalanced objects (as familiar and novel) across animals to preclude a possible bias towards a certain object that could contaminate the results. As an additional control, we performed another separate preference control experiment. Five new animals took part in this experiment. The procedure consisted of arena familiarization and test phases and there was no object familiarization phase. After three days of arena familiarization, the animals were given five minutes to enter into the arena and explore two different objects made of Lego. After this test phase, the control experiment was concluded and the animals were returned to their home cages.

\subsection{Data analyses and statistics}

Videos recorded during the object familiarization and test phases were analyzed offline to determine the time every animal explored each object. Pointing of the nose at an object at a distance of less than one $\mathrm{cm}$ or touching an object were considered criteria for object exploration. We determined both the time spent exploring each object and the total object exploration time. To analyze animals' novelty preference, we compared novel and familiar object exploration times. In addition, a discrimination index (DI) was calculated as the difference in time exploring novel and familiar objects divided by total exploration time.

$\mathrm{DI}[\%]=\frac{(\mathrm{NE}-\mathrm{FE})}{(\mathrm{NE}+\mathrm{FE})} \times 100$

where NE and FE refer to novel object exploration and familiar object exploration times respectively. To have an index for comparison, we also calculated a hypothetical discrimination index for the object familiarization phase using the same formula. In this case, the familiar object that was later to be replaced with the novel object served as the hypothetical novel object. All data are expressed as mean \pm SEM. Statistical analysis used one-sample $t$ tests, student's paired $t$-tests and repeated measures analysis of variance (ANOVA) followed by Tukey's and Newman-Keuls post hoc tests for multiple comparisons. A probability level less than 0.05 was considered as statistical significance.

\section{Results}

Before describing the results of the novel object recognition (NOR) tests, we present the results of a control experiment conducted to ensure that animals had no intrinsic preference among pairs of objects used in the NOR task. This control experiment was conducted in five animals that all were naïve to any behavioral experiment including the NOR test. A paired $t$-test showed that animals spent similar amount of time $\left(t_{\mathrm{obj} 1}=18.8 \pm 5.4 \mathrm{~s}\right.$, $t_{\mathrm{obj} 2}=19.1 \pm 5.8 \mathrm{~s}$ ) to explore each of the two different objects when they had no familiarization to either of these two objects $\left(t_{4}=-0.29\right.$, $p=0.78$ ). This suggests that the results observed in the NOR tests below are in fact due to recognition memory following familiarization and not artifacts resulting from our choice of object stimuli.

\subsection{Experiment 1}

This experiment examined recognition memory by means of novel object preference in tree shrew using a 24-h retention period and a single session of exposure to familiar objects. We found that animals spent a similar amount of time $\left(t_{\mathrm{obj} 1}=20.2 \pm 5.4 \mathrm{~s}\right.$, $t_{\mathrm{obj} 2}=17.1 \pm 2.9 \mathrm{~s}$ ) exploring each of the two identical objects during the object familiarization phase, demonstrating no preference for one of the objects or locations (paired $t$-test; $t_{4}=0.54, p=0.62$ ). Considering the total exploration time as a measure of animals' motivation for object exploration, animals' total exploration during the object familiarization and test phases was compared using a paired $t$-test. The comparison showed there was no significant difference in total exploration time $\left(t_{\text {total-fam }}=37.3 \pm 6.7 \mathrm{~s}\right.$, $\left.t_{\text {total-test }}=31.5 \pm 6.5 \mathrm{~s}\right)$ between object familiarization and test phase $\left(t_{4}=1.43 ; p=0.23 ;\right.$ Fig. $\left.2 \mathrm{~A}\right)$. To examine whether there was nevertheless any memory trace of having previously explored the familiar object, we tested if there was a significant reduction in exploration time of the familiar object between the object familiarization and test phases. Thus, the average time spent exploring each of the familiar objects during the object familiarization phase $(t=18.7 \pm 3.3 \mathrm{~s})$ was compared with the time spent exploring the familiar object during the test phase $(t=11.8 \pm 2.8 \mathrm{~s})$. A paired $t$-test showed that there was indeed significant reduction in familiar object exploration $\left(t_{4}=3.53, p=0.02\right.$; Fig. 2B). During the test phase, a reduction in motivation to explore the familiar object (i.e. partial habituation) was thus accompanied by an increased motivation to explore a newly included novel object, leading to overall unchanged exploration time as compared to the object familiarization phase. To examine the differences between exploration of novel $\left(t_{\text {novel }}=19.7 \pm 4.2 \mathrm{~s}\right)$ and familiar $\left(t_{\mathrm{fam}}=11.8 \pm 2.8 \mathrm{~s}\right)$ objects on the test day, a paired $t$-test on familiar and novel objects' 
(A) Total object exploration

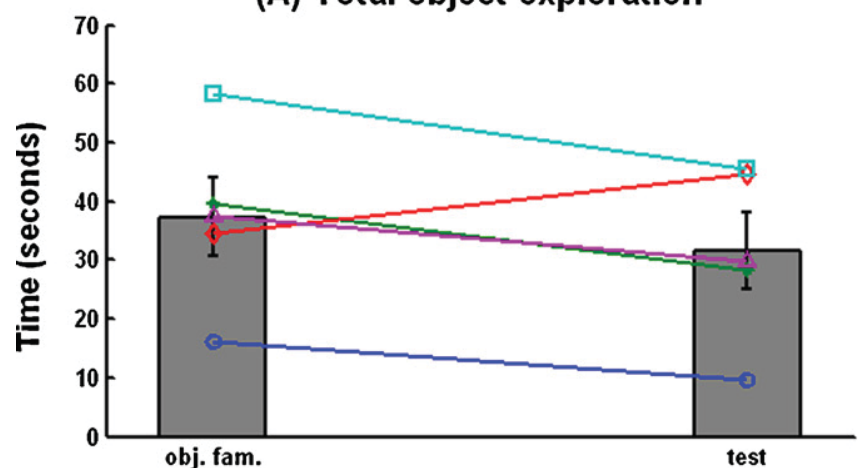

(C) Familiar and Novel object exploration

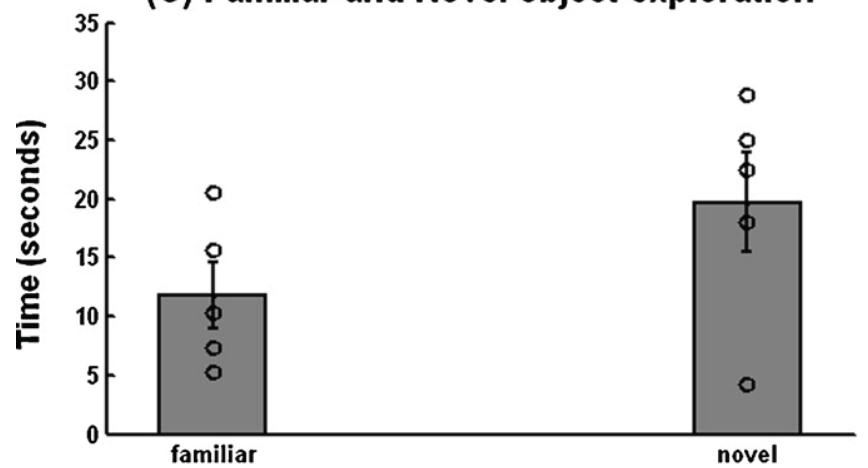

(B) Familiar object exploration

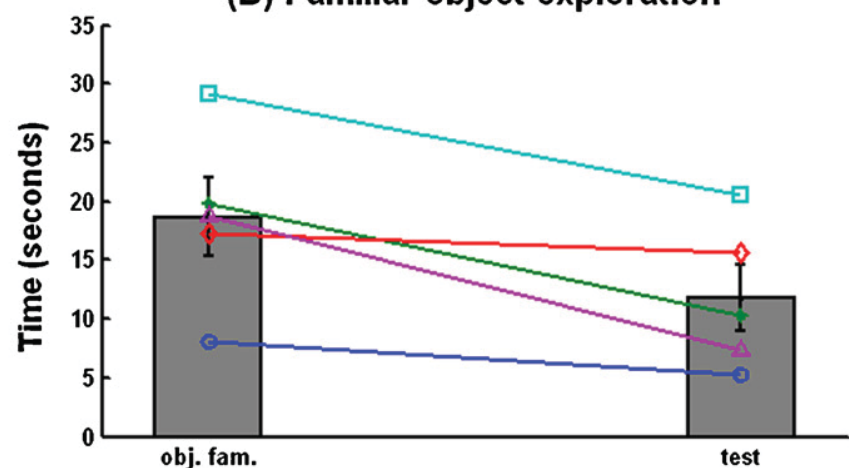

(D) Exploration time correlations

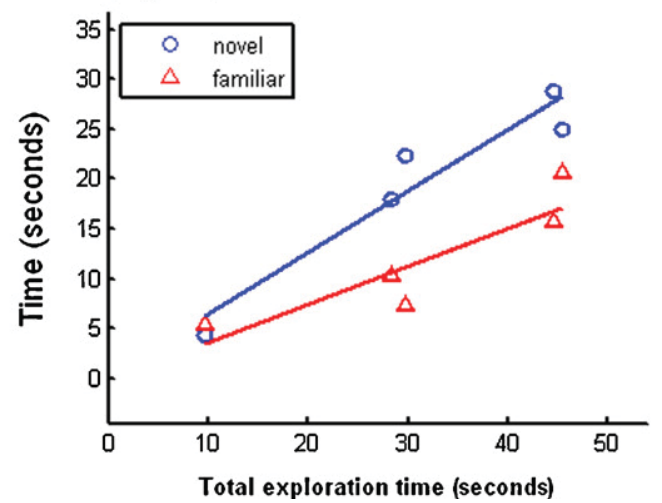

Fig. 2. Results of experiment 1. (A) Total exploration on the test and object familiarization days. There was no significant difference between total exploration on the object familiarization and test days. (B) Familiar object exploration on the object familiarization and on test days. (C) Novel and familiar object exploration on the test day. (D) Novel object exploration showed fairly strong linear correlation to the total exploration on the test day. Each symbol in A and B and each circle in C corresponds to one animal. Obj. fam. $=$ object familiarization.

exploration times revealed a non-significant trend towards exploration preference for the novel object during the test phase $\left(t_{4}=-2.71, p=0.053\right.$; Fig. 2C).

Finally, given the remarkable variability in total exploration among animals, we examined how much time individual animals spent exploring novel and familiar objects as a function of total exploration time (Fig. 2D). We found that both novel and familiar object exploration times increased with increasing total exploration time. Linear regression showed that novel object exploration amounted to roughly $62 \%$ of total exploration after a $24 \mathrm{~h}$ retention period following the object familiarization session $(c=0.618$, $r^{2}=0.915, p=0.0108$ ). Thus, despite the large variation of total exploration time among animals, a constant fraction of this time was spent exploring the novel object.

\subsection{Experiment 2}

Given the relatively weak 24-h memory retention in the first experiment, we proceeded to examine whether repeated exposure to familiar objects during multiple object familiarization sessions on consecutive days can enhance novelty preference during the test phase. This experiment was conducted twice (Experiments 2a and 2b) using two different sets of objects to rule out stimulus specific confounds. Similar to experiment 1 , animals spent a similar amount of time (Experiment 2a: $t_{\mathrm{obj} 1}=10.2 \pm 2.2 \mathrm{~s}, t_{\mathrm{obj} 2}=9.7 \pm 2.3 \mathrm{~s}$; and Experiment 2b: $t_{\mathrm{obj} 1}=17.4 \pm 2.8 \mathrm{~s}, t_{\mathrm{obj} 2}=17.1 \pm 2.5 \mathrm{~s}$ ) exploring each of the familiar objects during the object familiarization phase $\left(t_{6}=0.18, p=0.86\right.$; and $t_{5}=0.16, p=0.88$ for experiments $2 \mathrm{a}$ and $2 \mathrm{~b}$ respectively). Animals' total exploration during the object familiarization and test phase was compared using repeated measures one-way ANOVAs followed by Newman-Keuls post hoc test for multiple comparisons. This revealed a significant difference between the total exploration time $\left(t_{\text {total-fam } 1}=31.7 \pm 7.3 \mathrm{~s}\right.$ and $\quad 52.6 \pm 8.0 \mathrm{~s}, \quad t_{\text {total-fam } 2}=15.9 \pm 3.6 \mathrm{~s} \quad$ and $\quad 25.7 \pm 4.6 \mathrm{~s}$, $t_{\text {total-fam } 3}=12 \pm 2.9 \mathrm{~s}$ and $25.2 \pm 5.2 \mathrm{~s}, \quad t_{\text {total-test }}=27.0 \pm 5.0 \mathrm{~s}$ and $33.4 \pm 5.8 \mathrm{~s}$ for three days of object familiarization and the test day, $F(3,27)=4.88, p=0.012$ and $F(3,23)=9.97, p<0.001$, for experiments $2 \mathrm{a}$ and $2 \mathrm{~b}$ respectively; see Fig. $3 \mathrm{~A}$ ). Post hoc tests revealed that there was a significant difference between the total exploration time on the first day of object familiarization as compared with the total exploration on the second $(p<0.05 ; p<0.001$, respectively for $2 \mathrm{a}$ and $2 \mathrm{~b}$ ) and on the third ( $p<0.05 ; p<0.01$, respectively for $2 \mathrm{a}$ and $2 \mathrm{~b}$ ) days of object familiarization. This shows that on consecutive days of object familiarization, animals show a diminished motivation for familiar object exploration. Despite an increase for some animals on the test day, the total exploration time did not significantly differ between the third object familiarization day and the test day. Thus, the increased motivation for object exploration due to the inclusion of a novel object could not compensate for the prolonged habituation developed during successive days of object familiarization (Fig. 3A) Additionally, to examine the time course of reduction in exploration time of familiar objects throughout the object familiarization phase, the average of time spent exploring a familiar object during the object familiarization phase was compared to the familiar object exploration time during the test phase. We found that familiar object exploration time generally decreased with repeated exposure to the familiar object on subsequent days. Repeated measures one-way ANOVAs followed by Tukey's post hoc test for multiple comparisons revealed that there was a significant reduction in exploration time of familiar objects 
(A) Total object exploration

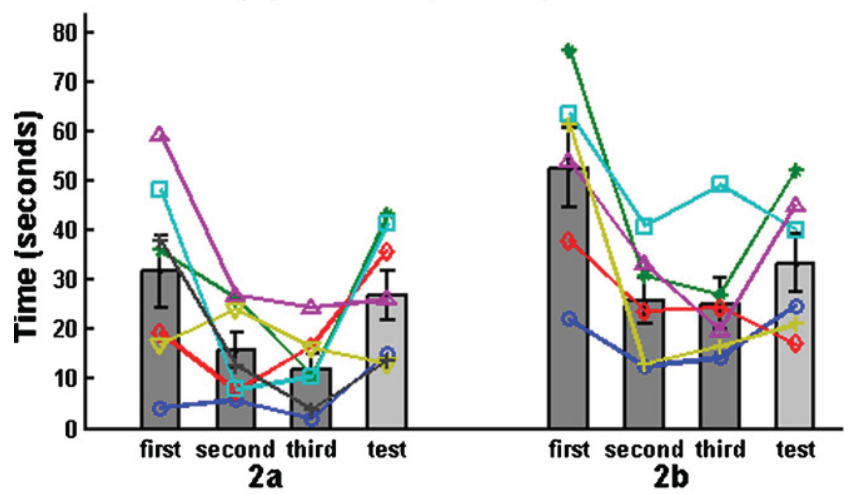

(C) Familiar and novel object exploration

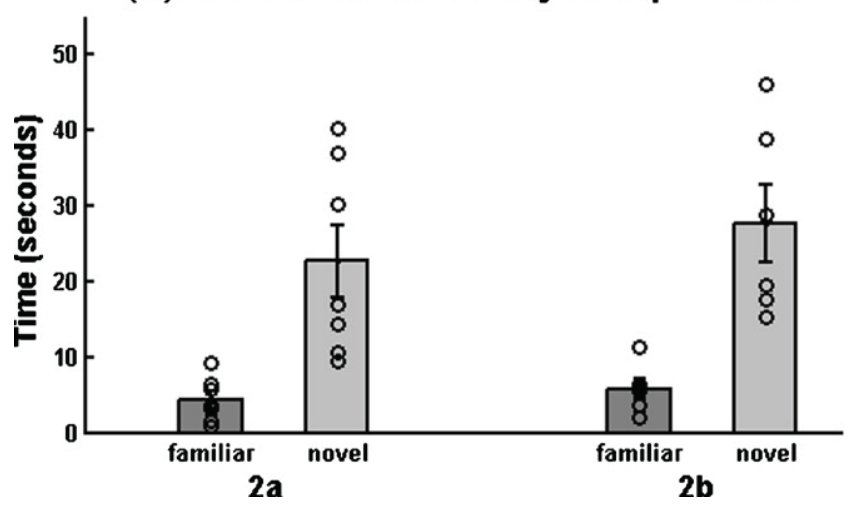

(B) Familiar object exploration

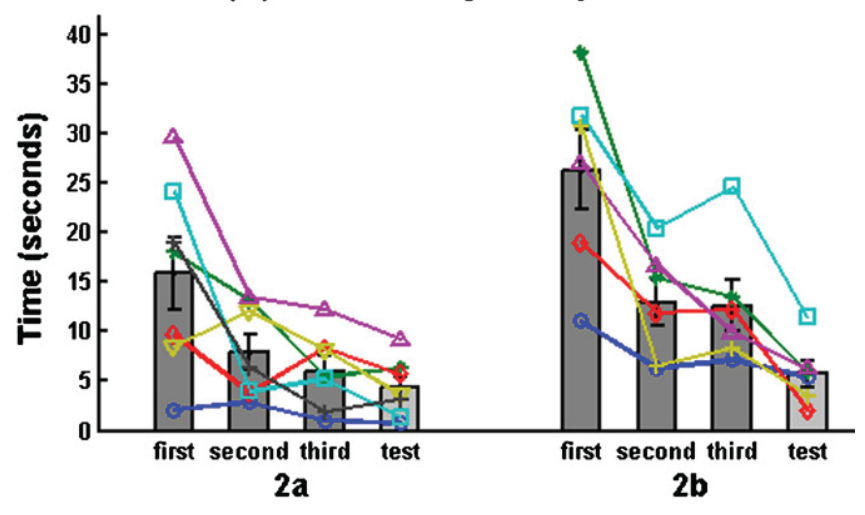

(D) Exploration time correlations

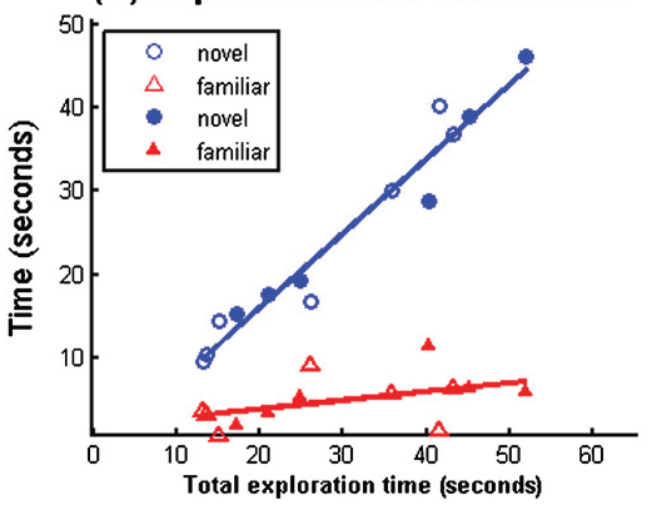

Fig. 3. Results of experiment 2 (2a and 2b). (A) Total exploration during the object familiarization phase (three days) and test phase (one day). (B) Familiar object exploration on three consecutive days of object familiarization and on the test day. (C) Novel and familiar object exploration on the test day. (D) Novel object exploration showed a strong linear correlation to the total exploration on the test day. Each symbol in A and B and each circle in C corresponds to one animal. In D, solid circles and triangles correspond to experiment 2 b. 'first', 'second' and 'third' refer to days of object familiarization.

$(F(3,27)=8.55, p=0.001 ; F(3,23)=19.1, p<0.0001$, respectively for $2 \mathrm{a}$ and $2 \mathrm{~b}$; Fig. 3B). Post hoc Tukey tests showed that the significant reduction in exploration time occurred just after the first day of object familiarization and there was no significant difference between days 2 and 3 of object familiarization and the test day in both experiments (or repetitions; Fig. 3B). Thus, the strongest reduction in familiar object exploration time occurred between the first and second object familiarization days.

To examine novelty preference on the test day, paired t-tests were employed on novel and familiar object exploration times. We found that animals spent significantly more time exploring the novel object compared to the familiar object (Experiment $2 \mathrm{a}: t_{\mathrm{fam}}=4.3 \pm 1.1 \mathrm{~s}, t_{\text {novel }}=22.6 \pm 4.8 \mathrm{~s}, t_{6}=-3.718, p<0.01$; Experiment $2 \mathrm{~b}: t_{\mathrm{fam}}=5.7 \pm 1.3 \mathrm{~s}, t_{\text {novel }}=27.7 \pm 5.1 \mathrm{~s}, t_{5}=-4.64, p<0.01$, respectively for $2 \mathrm{a}$ and $2 \mathrm{~b}$; Fig. $3 \mathrm{C}$ ). Thus, after three days of repeated exposure to familiar objects, tree shrew demonstrated robust recognition memory revealed by robust preference for novel object exploration.

Finally, we examined what fraction of the total exploration time individual animals spent on novel object exploration. To explore this, novel and familiar object exploration times were plotted against total exploration time (Fig. 3D). Linear regression showed that there was a strong correlation between novel object and total exploration time during the test phase $\left(c=0.84, r^{2}=0.95\right.$, $p<0.0001$ ). On average, animals thus spent about $84 \%$ of the total exploration time exploring the novel object. By contrast, familiar object exploration was only weakly correlated with total exploration time, and animals spent on average only about five seconds exploring the familiar object.

\subsection{Discrimination index}

Since the discrimination index is a commonly used measure for novelty preference, we calculated discrimination indexes for all experiments. A one-sample $t$-test showed that there was no significant discrimination between two different objects during the control experiment $\left(t_{4}=0.31, p=0.77\right)$ in the absence of object familiarization. In both experiments 1 and 2, during object familiarization, the discrimination index (familiar vs. hypothetical novel object) was also not significantly different from zero (one sample $t$-tests; $p=0.81, p=0.88, p=0.96$, respectively for experiments 1 , $2 \mathrm{a}$ and $2 \mathrm{~b}$; Fig. 4). For experiment 1, a one sample t-test showed that the discrimination index between the novel and the familiar object did not significantly differ from zero ( $p=0.105$; Fig. 4A). However, the discrimination index calculated for experiments $2 \mathrm{a}$ and $2 \mathrm{~b}$ revealed that animals exhibited a significant preference for the novel object ( $p<0.001 ; p<0.0001$, respectively; Fig. 4B). The discrimination index thus yielded similar results to the above analyses based on exploration time, providing further evidence for robust novelty preference in tree shrew following repeated object familiarization sessions.

\section{Discussion}

This study examined long term recognition memory in tree shrew using the novel object recognition test as a model test of spontaneous recognition memory without training. The experiments demonstrated that tree shrews exhibit recognition memory as shown by a robust novelty preference following three-day, but 
(A) Experiment 1

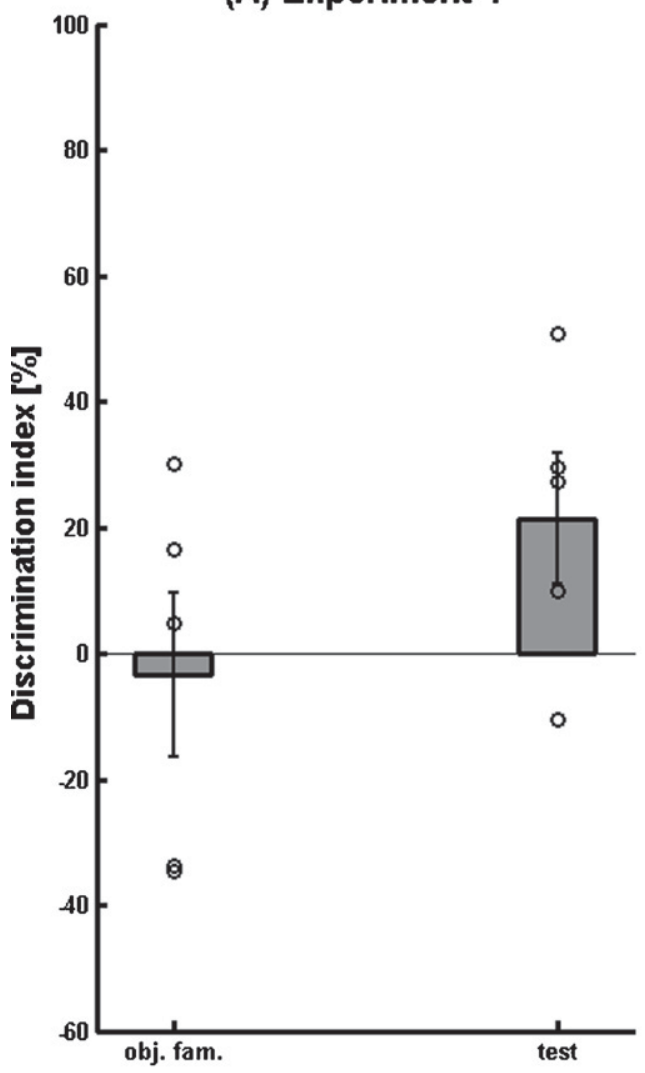

(B) Experiment 2

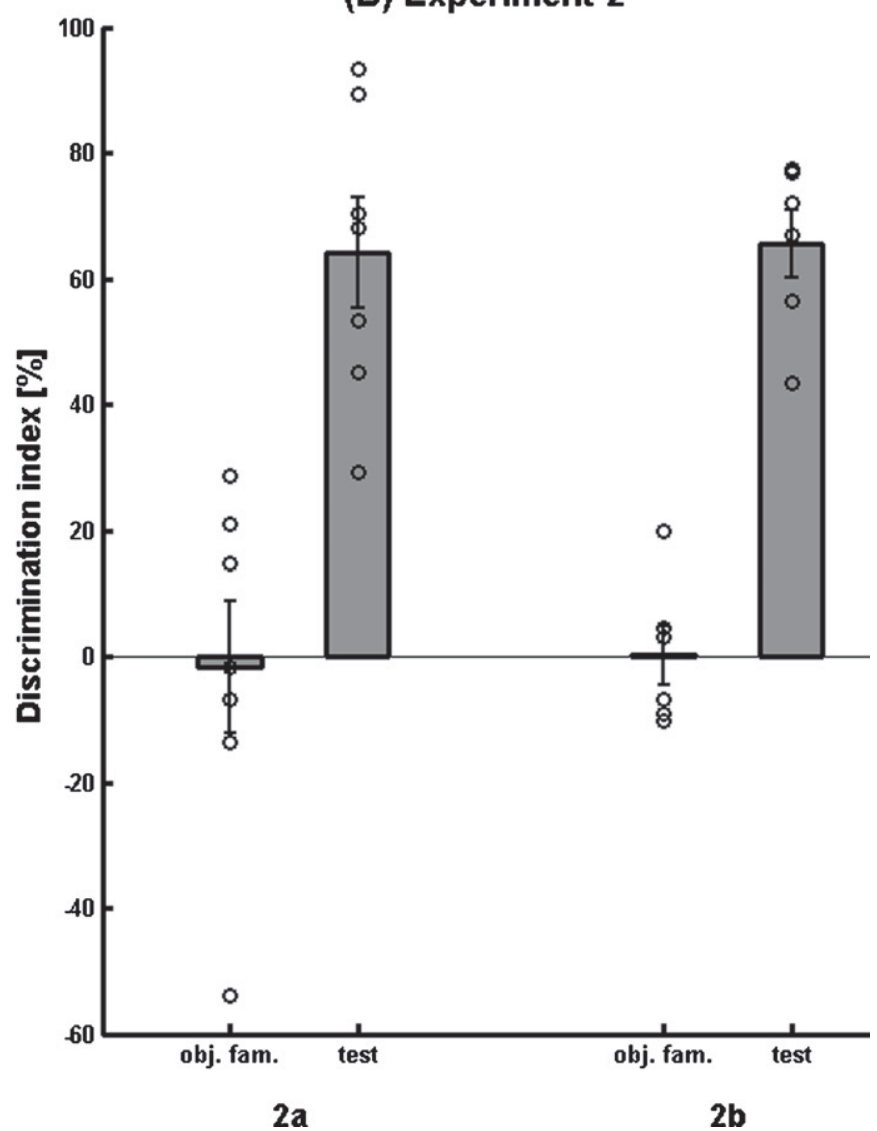

Fig. 4. Discrimination index in experiments 1 and 2. Hypothetical discrimination between two identical objects from familiarization phase was plotted for all experiments as a reference for discrimination between novel and familiar objects on the test day. Positive values on Y axis indicate novelty preference. (A) Discrimination index for experiment 1. (B) Discrimination index for experiment 2. Obj. fam. = object familiarization.

not one-day, familiarization to the familiar objects. In a one-day object familiarization paradigm, tree shrews showed some moderate evidence for recognition memory formation, as revealed by significant reduction in exploration time of the familiar object after a 24-h delay following object familiarization. Repeated exposure to the same familiar objects has been used relatively infrequently in the NOR paradigm (Save et al., 1992; Weible et al., 2009), and these previous experiments did not specifically address effects of repeated exposure on memory consolidation. This simple modification in the protocol in our experiment led to a robust discrimination between the familiar and novel object, reflecting a very vivid memory of the familiar object. Contrasting results of novelty preference in three-day and one-day object familiarization paradigms of the task demonstrates that repeated exposure to familiar objects significantly consolidates long term memory. This finding is in contrast to the suggestion of Gaskin et al. (2010) that increased exposure to familiar objects does not lead to an increased novelty preference. A species difference in exploratory behavior between tree shrew and rodents might account for these divergent results. Additionally, a methodological difference between the studies might also play a role: While Gaskin et al. repeatedly used the same familiar object, coupled with different new objects, in subsequent test sessions to increase familiarity, we used multiple object familiarization days with the same familiar objects for this purpose. Thus, prolonged object familiarization in our experiments could efficiently consolidate memory of the familiar object, while using the same familiar object in subsequent testing sessions, as in the Gaskin et al. study, might have failed to consolidate this memory due to interference from a new object on every subsequent test trial.
Our experiments showed that while reduction in familiar objects exploration (i.e. habituation) occurs over successive days of exposure, the strongest habituation takes place following the first object familiarization session in both one-day and three-day object familiarization paradigms of long term NOR task. Despite the lack of significant novelty preference in the one-day object familiarization paradigm of the task, significant reduction in familiar object exploration time emerges on the test day already after a one-day object familiarization phase. One may argue that reduced exploration of the familiar object is due to the presence of a novel object rather than habituation to the familiar object per se. However, a significant habituation to the familiar object also occurred in a three-day object familiarization paradigm following the first day of object familiarization. Since this significant habituation was evident on the second day of object familiarization in the absence of a novel object, it follows that the process of habituation to familiar objects develops following a one-session exposure to these objects independently from the presence of a novel object. Taken together, the strongest habituation to the familiar objects occurred following the first day of object familiarization, while subsequent object familiarization days resulted in relatively weak additional habituation.

In agreement with the original work on the NOR task (Ennaceur and Delacour, 1988), our experiment showed that despite the lack of a significant difference in total exploration in the one-day object familiarization paradigm of the task, significant habituation does occur to the familiar object. While habituation effects were strongest after the first day of object familiarization, robust novelty preference only occurred after multiple familiarization days with familiar objects, i.e. significant habituation to familiar object is 
not sufficient to elicit significant novelty preference, but a stronger degree of habituation,which lies well above significance, is required to produce robust novelty preference. We suggest that during the second and third object familiarization days, animals further explore the familiar objects, resulting in a consolidated representation in long-term memory. A well-consolidated representation of the familiar object is a prerequisite for observing robust novelty preference in the NOR task. It is thus possible that the objects are already encoded and stored in long-term memory during the first visual encounter. However, in order for the encoded information to be easily available for comparison in the presence of a novel object, the encoded information needs to be consolidated by further familiarization sessions. In the context of novel object recognition, the three phenomena of memory consolidation, habituation and novelty preference are strongly inter-related and cannot be discussed independent of each other. It should be noted that the notion of novelty preference in this task inevitably entails the existence of an already familiar object that has lost its novelty to the animal, i.e. has been habituated. So, we suggest that habituation and novelty preference are indications of memory and are behavioral processes accompanying the formation of recognition memories.

Despite remarkable individual differences in total exploration amongst animals, we observed a strong linear correlation between total exploration and novel object exploration indicating a roughly constant percentage of novelty preference among animals in each experiment. This indicates that individual differences in total exploration influence the degree of novel object exploration in a proportionate manner, leaving the ratio of novel object exploration time to total exploration time unchanged. These individual differences may result from variation of exploratory motivation among animals or alternatively from differences in the dynamics of object processing. However, regardless of the cause of individual differences, the proportion of novelty preference remains constant. The striking linearity of novel object exploration as a function of total exploration suggests that the degree of novelty preference in both one-day and three-day object familiarization paradigms of the experiment remains constant and is not influenced by the general motivation of animal for exploration or differential processing dynamics. This suggests that individual differences in exploratory motivation among animals result from changes in motivational brain circuits that are largely independent from the circuits involved in object memory.

Most previous behavioral studies in tree shrews have used rewards like sugared water, almond or food to motivate animals for task participation (Callahan and Petry, 2000; Ohl et al., 1998; Petry et al., 1984). To our knowledge, our study is the first to demonstrate behavioral preference effects in tree shrew based on intrinsic motivation in the absence of external rewards. Whether such effects could be demonstrated had been questionable due to the fast-moving and stress-vulnerable characteristics of these animals when exposed to new environments. We overcame this limitation by using an arena closely resembling the animals' home cage, combined with the use of nesting box connected to the arena providing an open two-way pathway to the nesting box. This setup provides a situation to test animals' exploratory behavior while minimizing animals' level of stress at the same time.

Previous work has shown that rodents exhibit robust novelty preference following short-term retention intervals. However, there are inconsistent results in the literature regarding long-term memory retention effects in rodents: While many studies have shown a lack of significant retention in a long term $(24 \mathrm{~h})$ memory NOR task (Ennaceur and Delacour, 1988; Okuda et al., 2004; Roncarati et al., 2009; Tellez et al., 2010), a few recent studies have demonstrated such long-term retention effects (Botton et al., 2010; Reger et al., 2009). This disparity is most likely due to differences in methodological nuances like the degree of object familiarization, the manner of handling animals or the animals' environment and their level of stress. However, most of the studies of this kind did not discuss the reasons accounting for the lack of LTM in the NOR task and focused on the effects of certain treatments on memory. Note however, that the above studies in rodents generally used a single object familiarization session. An examination of rodent behavior in the NOR task using multiple object familiarization sessions is required to address whether tree shrews are indeed more effective in terms of encoding memories for objects than rodents, as our findings suggest. Nonetheless, an immediate advantage of tree shrew over rodents is its phylogenetic closeness to humans despite having almost the same size as the rat. So, in addition to filling the gap between rodents and primates for comparative studies, the tree shrew is increasingly put forward as a potential candidate as a model for studies of both brain disorders and basic understanding of brain function (Cao et al., 2003).

The visual paired comparison or preferential looking tasks are closely related to the NOR paradigm, and have been used to test recognition memory in human infants and non-human primates. Using these tasks, novelty preference has been demonstrated following short-term retention periods (Bachevalier and Nemanic, 2008; Pascalis and de Schonen, 1994; Jutras and Buffalo, 2010; Zeamer et al., 2010) in both human infants and monkeys. A number of studies have also documented novelty preference following long-term retention intervals (Gunderson and Swartz, 1985; Pascalis et al., 1998). In addition, the effect of familiarization time and re-familiarization on novelty preference has been studied in human and non-human primates (Gunderson and Swartz, 1986; Monk et al., 1996; Morgan and Hayne, 2006). Available evidence suggests that generally increased familiarization and refamiliarization result in increased novelty preference. In particular, Monk et al. (1996) showed that only infant monkeys, who had a re-familiarization trial preceding the test trial in visual pairedcomparison test exhibited novelty responses. This similarity in the effect of re-familiarization on visual recognition memory between tree shrew and monkey infants is consistent with the close phylogenetic relationship between these species and suggests tree shrews as attractive model animals for further recognition memory investigations.

\section{Acknowledgements}

This work was supported by EURYI grant PE0033-117106 and by the University of Fribourg. The authors would like to thank P. Lavanex and E. Fuchs for helpful comments on the earlier versions of this manuscript, as well as R. Kretz for helpful advice.

\section{References}

Aubele, T., Kaufman, R., Montalmant, F., Kritzer, M.F., 2008. Effects of gonadectomy and hormone replacement on a spontaneous novel object recognition task in adult male rats. Horm. Behav. 54, 244-252.

Bachevalier, J., Nemanic, S., 2008. Memory for spatial location and object-place associations are differently processed by the hippocampal formation, parahippocampal areas TH/TF and perirhinal cortex. Hippocampus 18, 64-80.

Botton, P.H., Costa, M.S., Ardais, A.P., Mioranzza, S., Souza, D.O., da Rocha, J.B., Porciúncula, L.O., 2010. Caffeine prevents disruption of memory consolidation in the inhibitory avoidance and novel object recognition tasks by scopolamine in adult mice. Behav. Brain Res. 214, 254-259.

Callahan, T.L., Petry, H.M., 2000. Psychophysical measurement of temporal modulation sensitivity in the tree shrew (Tupaia belangeri). Vision Res. 40, 455-458.

Cao, J., Yang, E.B., Su, J.J., Li, Y., Chow, P., 2003. The tree shrews: adjuncts and alternatives to primates as models for biomedical research. J. Med. Primatol. 32, 123-130.

Donkin, J.J., Stukas, S., Hirsch-Reinshagen, V., Namjoshi, D., Wilkinson, A., May, S., Chan, J., Fan, J., Collins, J., Wellington, C.L., 2010. ATP-binding cassette transporter A1 mediates the beneficial effects of the liver-X-receptor agonist GW3965 on object recognition memory and amyloid burden in APP/PS1 mice. J. Biol. Chem. 285, 34144-34154.

Ennaceur, A., Delacour, J., 1988. A new one-trial test for neurobiological studies of memory in rats 1: behavioral data. Behav. Brain Res. 31, 47-59. 
Fahey, B., Barlow, S., Day, J.S., O'Mara, S.M., 2008. Interferon-alpha-induced deficits in novel object recognition are rescued by chronic exercise. Physiol. Behav. 95, $125-129$.

Fuchs, E., 2005. Social stress in tree shrews as an animal model of depression: an example of a behavioral model of a CNS disorder. CNS Spectrums 10, 182-190.

Fuchs, E., Flügge, G., 2003. Chronic social stress: effects on limbic brain structures. Physiol. Behav. 79, 417-427.

Fuchs, E., Flügge, G., 2002. Social stress in tree shrews: effects on physiology, brain function, and behavior of subordinate individuals. Pharmacol. Biochem. Behav. 73, 247-258.

Gaskin, S., Tardif, M., Cole, E., Piterkin, P., Kayello, L., Mumby, D.G., 2010. Object familiarization and novel-object preference in rats. Behav. Process 83, 61-71.

Greco, S.J., Bryan, K.J., Sarkar, S., Zhu, X., Smith, M.A., Ashford, J.W., Johnston, J.M., Tezapsidis, N., Casadesus, G., 2010. Leptin reduces pathology and improves memory in a transgenic mouse model of Alzheimer's disease. J. Alzheimers Dis. $19,1155-1167$

Gunderson, V.M., Swartz, K.B., 1985. Visual recognition in infant pigtailed macaques after a 24-hour delay. Am. J. Prim. 8, 259-264.

Gunderson, V.M., Swartz, K.B., 1986. Effects of familiarization time on visual recognition memory in infant pigtailed macaques (Macaca nemestrina). Dev. Psychol $22,477-480$.

Hauser, T.A., Kucinski, A., Jordan, K.G., Gatto, G.J., Wersinger, S.R., Hesse, R.A., Stachowiak, E.K., Stachowiak, M.K., Papke, R.L., Lippiello, P.M., Bencherif, M., 2009. TC-5619: an alpha7 neuronal nicotinic receptor-selective agonist that demonstrates efficacy in animal models of the positive and negative symptoms and cognitive dysfunction of schizophrenia. Biochem. Pharmacol. 78, 803-812.

Hillen, H., Barghorn, S., Striebinger, A., Labkovsky, B., Müller, R., Nimmrich, V., Nolte, M.W., Perez-Cruz, C., van der Auwera, I., van Leuven, F., van Gaalen, M., Bespalov, A.Y., Schoemaker, H., Sullivan, J.P., Ebert, U., 2010. Generation and therapeutic efficacy of highly oligomer-specific beta-amyloid antibodies. J. Neurosci. 30, 10369-10379.

Jurdak, N., Kanarek, R.B., 2009. Sucrose-induced obesity impairs novel object recognition learning in young rats. Physiol. Behav. 96, 1-5.

Jutras, M.J., Buffalo, E.A., 2010. Recognition memory signals in the macaque hippocampus. Proc. Natl. Acad. Sci. U. S. A. 107, 401-406.

Kozicz, T., Bordewin, L.A., Czéh, B., Fuchs, E., Roubos, E.W., 2008. Chronic psychosocial stress affects corticotropin-releasing factor in the paraventricular nucleus and central extended amygdala as well as urocortin 1 in the non-preganglionic Edinger-Westphal nucleus of the tree shrew. Psychoneuroendocrinology 33, 741-754.

Lockrow, J., Boger, H., Bimonte-Nelson, H., Granholm, A.C., 2010. Effects of longterm memantine on memory and neuropathology in Ts65Dn mice, a model for Down syndrome. Behav. Brain Res., http://dx.doi.org/10.1016/j.bbr.2010.03.036, 251658240.

Lu, H.D., Petry, H.M., 2003. Temporal modulation sensitivity of tree shrew retinal ganglion cells. Vis. Neurosci. 20, 363-372.

Lu, P., Mamiya, T., Lu, L.L., Mouri, A., Zou, L., Nagai, T., Hiramatsu, M., Ikejima, T., Nabeshima, T., 2009. Silibinin prevents amyloid beta peptide-induced memory impairment and oxidative stress in mice. Br. J. Pharmacol. 157, 1270-1277.

Martin, R.D., 1968. Towards a new definition of primates. Man 3, 377-401.

McLean, S.L., Idris, N.F., Woolley, M.L., Neill, J.C., 2009. D(1)-like receptor activation improves PCP-induced cognitive deficits in animal models: Implications for mechanisms of improved cognitive function in schizophrenia. Eur. Neuropsychopharmacol. 19, 440-450

Monk, C.S., Gunderson, V.M., Grant, K.S., Mechling, J.L., 1996. A demonstration of the memory savings effect in infant monkeys. Dev. Psychol. 32, 1051-1055.

Morgan, K., Hayne, H., 2006. The effect of encoding time on retention by infants and young children. Infant Behav. Dev. 29, 599-602.

Mouri, A., Noda, Y., Hara, H., Mizoguchi, H., Tabira, T., Nabeshima, T., 2007. Oral vaccination with a viral vector containing Abeta cDNA attenuates age-related Abeta accumulation and memory deficits without causing inflammation in a mouse Alzheimer model. FASEB J. 21, 2135-2148.

Neill, J.C., Barnes, S., Cook, S., Grayson, B., Idris, N.F., McLean, S.L., Snigdha, S., Rajagopal, L., Harte, M.K., 2010. Animal models of cognitive dysfunction and negative symptoms of schizophrenia: focus on NMDA receptor antagonism. Pharmacol. Ther. 128, 419-432.

Nishida, Y., Yokota, T., Takahashi, T., Uchihara, T., Jishage, K., Mizusawa, H., 2006. Deletion of vitamin E enhances phenotype of Alzheimer disease model mouse. Biochem. Biophys. Res. Commun. 350, 530-536.

Ohl, F., Fuchs, E., 1999. Differential effects of chronic stress on memory processes in the tree shrew. Cog. Brain Res. 7, 379-387.

Ohl, F., Oitzl, M.S., Fuchs, E., 1998. Assessing cognitive functions in tree shrews: visuo-spatial and spatial learning in the home cage. J. Neurosci. Methods 81, 35-40.

Ohta, H., Matsutani, S., Ishida, H., Matano, S., 1985. Learning set formation in common tree shrews (Tupaia glis). Folia Primatol 44, 204-209.

Okuda, S., Roozendaal, B., McGaugh, J.L., 2004. Glucocorticoid effects on object recognition memory require training-associated emotional arousal. Proc. Natl. Acad. Sci. U. S. A. $101,853-858$.

Ozawa, K., Hashimoto, K., Kishimoto, T., Shimizu, E., Ishikura, H., Iyo, M., 2006. Immune activation during pregnancy in mice leads to dopaminergic hyperfunction and cognitive impairment in the offspring: a neurodevelopmental animal model of schizophrenia. Biol. Psychiatry 59, 546-554.

Pascalis, O., de Haan, M., Nelson, C.A., de Schonen, S., 1998. Long-term recognition memory for faces assessed by visual paired comparison in 3- and 6-month-old infants. J. Exp. Psychol. Learn. Mem. Cogn. 24, 249-260.

Pascalis, O., de Schonen, S., 1994. Recognition memory in 3- to 4-day-old human neonates. Neuroreport 5, 1721-1724.

Petry, H.M., Fox, R., Casagrande, V.A., 1984. Spatial contrast sensitivity of the tree shrew. Vision Res. 24, 1037-1042.

Powell, K.J., Hori, S.E., Leslie, R., Andrieux, A., Schellinck, H., Thorne, M., Robertson, G.S., 2007. Cognitive impairments in the STOP null mouse model of schizophrenia. Behav. Neurosci. 121, 826-835.

Raczkowski, D., Casagrande, V.A., Diamond, I.T., 1976. Visual neglect in the tree shrew after interruption of the descending projections of the deep superior colliculus. Exp. Neurol. 50, 14-29.

Reger, M.L., Hovda, D.A., Giza, C.C., 2009. Ontogeny of rat recognition memory measured by the novel object recognition task. Dev. Psychobiol. 51, 672-678.

Roncarati, R., Scali, C., Comery, T.A., Grauer, S.M., Aschmi, S., Bothmann, H., Jow, B., Kowal, D., Gianfriddo, M., Kelley, C., Zanelli, U., Ghiron, C., Haydar, S., Dunlop, J., Terstappen, G.C., 2009. Procognitive and neuroprotective activity of a novel alpha7 nicotinic acetylcholine receptor agonist for treatment of neurodegenerative and cognitive disorders. J. Pharmacol. Exp. Ther. 329, 459-468.

Save, E., Poucet, B., Foreman, N., Buhot, M.C., 1992. Object exploration and reactions to spatial and nonspatial changes in hooded rats following damage to parietal cortex or hippocampal formation. Behav. Neurosci. 106, 447-456.

Taglialatela, G., Hogan, D., Zhang, W.R., Dineley, K.T., 2009. Intermediate- and longterm recognition memory deficits in Tg2576 mice are reversed with acute calcineurin inhibition. Behav. Brain Res. 200, 95-99.

Takahashi, M., Ushitani, T., Fujita, K., 2008. Inference based on transitive relation in tree shrews (Tupaia belangeri) and rats (Ratus norvegicus) on a spatial discrimination task. Psychol. Rec. 58, 215-227.

Tellez, R., Rocha, L., Castillo, C., Meneses, A., 2010. Autoradiographic study of serotonin transporter during memory formation. Behav. Brain Res. 212, 12-26.

Vigano, D., Guidali, C., Petrosino, S., Realini, N., Rubino, T., Di Marzo, V., Parolaro, D., 2009. Involvement of the endocannabinoid system in phencyclidine-induced cognitive deficits modelling schizophrenia. Int. J. Neuropharmacol. 12, 599-614.

Weible, A.P., Rowland, D.C., Pang, R., Kentros, C., 2009. Neural correlates of novel object and novel location recognition behavior in the mouse anterior cingulate cortex. J. Neurophysiol. 102, 2055-2068.

Wong, F.K., Lee, S.H., Atcha, Z., Ong, A.B., Pemberton, D.J., Chen, W.S., 2010. Rasagiline improves learning and memory in young healthy rats. Behav. Pharmacol. 21, $278-282$.

Zambello, E., Fuchs, E., Abumaria, N., Rygula, R., Domenici, E., Caberlotto, L., 2010. Chronic psychosocial stress alters NPY system: different effects in rat and tree shrew. Prog. Neuropsychopharmacol. Biol. Psychiatry 34, 122-130.

Zeamer, A., Heuer, E., Bachevalier, J., 2010. Developmental trajectory of object recognition memory in infant rhesus macaques with and without neonatal hippocampal lesions. J. Neurosci. 30, 9157-9165. 